at http: //www.unison. ie/irish_independent/

index.php3?issue_id $=10318$

Seenan, G. (2004) Hidden toll of a justice system in crisis. Guardian, 21 January 2004. Available at http: // www.guardian.co.uk/uk_news/story/0,3604,II27369, 00.html

Shaw, J., Baker, D., Hunt, I. M., et al (2004) Suicide by prisoners: national clinical survey. British Journal of Psychiatry, 184, 263-267.

B. D. Kelly, H. G. Kennedy National Forensic Psychiatry Service, Central Mental Hospital,

Dundrum, Dublin 14, Ireland.

E-mail: brendankelly35@hotmail.com

\section{Ethnicity and suicidality}

Gunnell et al's (2004) interesting study came with useful learning points. However, while known as important factors that influence development and amelioration of suicidal thoughts, ethnicity and religion were not included in the study. As the world has become a small, or big, village, and as we live in a multi-ethnic and multi-religious society, I feel this should be considered as an additional limitation to the study. The relationship between religion and suicide became famous through Durkheim's study in the 19th century.

In European countries, evidence suggests that the prevalence of suicide continues to vary in accordance with international differences in traditions, customs and religious practices (Cavanagh \& Masterton, 1998). Cavanagh \& Masterton suggested that the strength of these differences is decreasing because of homogenisation among countries. In my opinion, it is unlikely that this influence will completely disappear. In a modern secularised society, religion is still a meaningful and protective factor for many individuals in a suicidal crisis (Lonnqvist, 2000).

Makinen \& Wassermann (2001) believe that much of the difference in suicidal behaviour between national groups can be connected with differences in cultural outlook, and state that 'traditionally religion has been considered to be the matrix of culture'.

Various factors that influence development and amelioration of suicidal thoughts do not function separately. I wonder, had ethnicity and religion been included, how would this have affected the outcome?

Cavanagh, J.T. O. \& Masterton, G. (1998) Suicide and deliberate self-harm. In Companion to Psychiatric Studies (6th edn) (eds E. Johnstone, C. Freeman \& A. Zealley), pp. 75I-783. Edinburgh: Churchill Livingstone.
Gunnell, D., Harbord, R., Singleton, N., et al (2004) Factors influencing the development and amelioration of suicidal thoughts in the general population. Cohort study British Journal of Psychiatry, 185, 385-393.

Lonnqvist, J. K. (2000) Suicide: epidemiology and causes of suicide. In New Oxford Textbook of Psychiatry, vol. I (eds M. G. Gelder, J. J. López-lbor \& N. Andreasen) pp. 1033-1039. Oxford: Oxford University Press.

Makinen, I. H. \& Wasserman, D. (200I) Some social dimensions of suicide. In Suicide: An Unnecessary Death (ed. D.Wasserman), pp. I0I-108. London: Martin Dunitz.

M. El-AdI Princess Marina Hospital, Upton, Northampton NN5 6UH, UK

E-mail: mamdouh.eladı@nht.northants.nhs.uk

Authors' reply: We agree with Dr El-Adl's comment that both ethnicity and religion may influence the incidence of, and recovery from, suicidal thoughts. Data on ethnicity were collected in the Office for National Statistics Survey that formed the basis of our paper (Singleton et al, 2001). Because of the relatively small sample size, only $122(5.1 \%)$ of the individuals who reported ethnicity were from a Black or minority ethnic group and only seven of these experienced incident suicidal thoughts. Thus, specific investigation of the impact of belonging to a particular ethnic group was not possible. If the Black and minority groups are combined to give a single group, the odds ratio for incident suicidal thoughts in this group compared with the White group in analyses adjusted for age, gender and score on the Clinical Interview Schedule - Revised is 0.77 (95\% CI 0.27-2.17). The breadth of the confidence interval indicates that the data are compatible with either a threefold reduction or a doubling in risk. Data on religion were not collected in the Office for National Statistics Survey of Psychiatric Morbidity.

Singleton, N., Bumpstead, R., O'Brien, M., et al (200I) Psychiatric Morbidity among Adults Living in Private Households, 2000. London: Stationery Office.

D. Gunnell, R. Harbord Department of Social

Medicine, University of Bristol, Canynge Hall,

Whiteladies Road, Bristol BS8 2PR, UK.

E-mail: D. J.Gunnell@bristol.ac.uk

N. Singleton Social Survey Division, Office for National Statistics, London, UK

R. Jenkins WHO Collaborating Centre, Institute of Psychiatry, London, UK

G. Lewis Division of Psychiatry, Cotham House, University of Bristol, Bristol, UK

\section{Attention deficit disorder in adults}

The editorial on attention-deficit hyperactivity disorder and life-span development (McArdle, 2004) is timely. However, it highlights the issues from the perspective of clinicians who may be directly involved in treating the disorder.

In general adult psychiatry, however, it is not widely recognised that (adult) attention-deficit disorder (ADD) is not uncommon and that people presenting with diagnoses of psychotic disorders, mood disorders, anxiety disorders, etc., may also be suffering from unrecognised ADD. This has profound implications for both treatment and outcome. For example, if a person develops a hypomanic or manic episode superimposed on $\mathrm{ADD}$, it is possible that the clinician unaware of ADD may end up overtreating the mood episode, as the baseline ADD may mislead the clinician into believing that the talkativeness and hyperactivity (of ADD) are an indication of elevated mood. The consequences include higher than necessary doses of medications, combination pharmacotherapy and increased length of stay in hospital. In patients with schizophrenia it is possible that the impairments in functioning caused by independent ADD may potentiate the poor functioning caused by schizophrenia. Again, if ADD is not recognised, it is possible that the poor outcome may be attributed to 'resistant' or 'residual' schizophrenia or perhaps to poor motivation. It is important to assess comorbidity such as ADD at the very first contact with mental health services, and early intervention service providers are ideally placed for this.

Regarding treatment, new strategies (other than stimulant medications) need to be developed, as stimulants may have destabilising effects on the baseline mental illness.

McArdle, P. (2004) Attention-deficit hyperactivity disorder and life-span development. British Journal of Psychiatry, 184, 468-469.

P. Gangdev Tooting and Furzedown Community Mental Health Trust, Springfield University Hospital, 6I Glenburnie Road, London SWI7 7DJ, UK. E-mail: Prakash.Gangdev@swlstg-tr.nhs.uk

\section{Risk reduction studies in schizophrenia}

Niemi et al's (2004) report does not truly address the implications of their findings. Their clinical implications (p.16) 